\title{
Development of Solar Electricity Supply System in India: An Overview
}

\author{
Sandeep Kumar Gupta ${ }^{1}$ and Raghubir Singh Anand ${ }^{2}$ \\ ${ }^{1}$ Department of Industrial \& Management Engineering, Indian Institute of Technology Kanpur, Kanpur 208016, India \\ ${ }^{2}$ Department of Electrical Engineering, Indian Institute of Technology Kanpur, Kanpur 208016, India
}

Correspondence should be addressed to Sandeep Kumar Gupta; sandipkg@iitk.ac.in

Received 26 January 2013; Revised 26 March 2013; Accepted 2 April 2013

Academic Editor: Paulo Fernandes

Copyright ( 2013 S. K. Gupta and R. S. Anand. This is an open access article distributed under the Creative Commons Attribution License, which permits unrestricted use, distribution, and reproduction in any medium, provided the original work is properly cited.

\begin{abstract}
Solar electricity supply system has grown at very rapid pace in India during the last few years. A total of $1047.84 \mathrm{MW}$ of grid connected photovoltaic projects and 160.8 MW of off-grid systems have been commissioned under different policy mechanisms between January 2010 and November 2012. It is observed that solar capacity development has achieved a greater height under state policies $(689.81 \mathrm{MW})$ than others. A study is made in this paper of various national and state level schemes, incentives, packages, instruments, and different mechanisms to promote solar photovoltaics and its effectiveness.
\end{abstract}

\section{Introduction}

The changing lifestyle with rapid industrialization has made electricity an indispensable and essential commodity over the years. During the last few decades, increasing prices of electricity with increasing demand and decreasing fossil fuel reserves have raised many concerns for policy makers, investors, and customers. Moreover, existing supply chain also poses a challenge of carbon foot print due to its dependency on fossil fuels like coal and oil for electricity generation [1-3].

To alleviate the concern, policy makers across the world have been looking for some sustainable and feasible alternative input energy sources for electricity generation. They found many options like nuclear, wind, solar, hydro, biomass, tidal, geothermal, and so forth $[3,4]$. However, literature supports solar energy as it is the most ready and green option available across the world [5]. The report published by Indian Meteorological Department (IMD), Ministry of Earth Sciences, Government of India (GoI) [6] states that

The solar energy received by the earth is more than 15,000 times the world's commercial energy consumption and over 100 times the world's known coal, gas and oil reserves. And this energy is readily available during the day for anyone to tap and that too free and without any constraint.
Initially used to supply electricity to satellites due to its high generation cost, solar technologies and its potential have improved enough to supply electricity not only to remote locations but also to supplement the national grid power at multimegawatt levels [7].

In India, wind- and solar-based systems have been getting good response under the conducive environment created through different policy measures. In this paper, our focus is only on the development of solar-based electricity supply systems. There are different kinds of support measures to promote grid connected and off-grid solar systems. We discuss these in detail in the subsequent sections.

\section{India's Potential for Solar Energy}

India is located in the northern hemisphere, lying between latitudes $8^{\circ} 4^{\prime} \mathrm{N}$ and $37^{\circ} 6^{\prime} \mathrm{N}$ and longitudes $68^{\circ} 7^{\prime} \mathrm{E}$ and $97^{\circ} 25^{\prime} \mathrm{E}$; the country is divided into almost two equal halves by the Tropic of Cancer $\left(23^{\circ} 30^{\prime} \mathrm{N}\right)$. The southern half which coincides with peninsular India lies in the tropical zone, while the northern half belongs to the subtropical zone.

Due to its locational advantage, on average, the country experiences 250 to 300 sunny days per year and receives an average hourly radiation of $200 \mathrm{MW} / \mathrm{km}^{2}$. The annual 


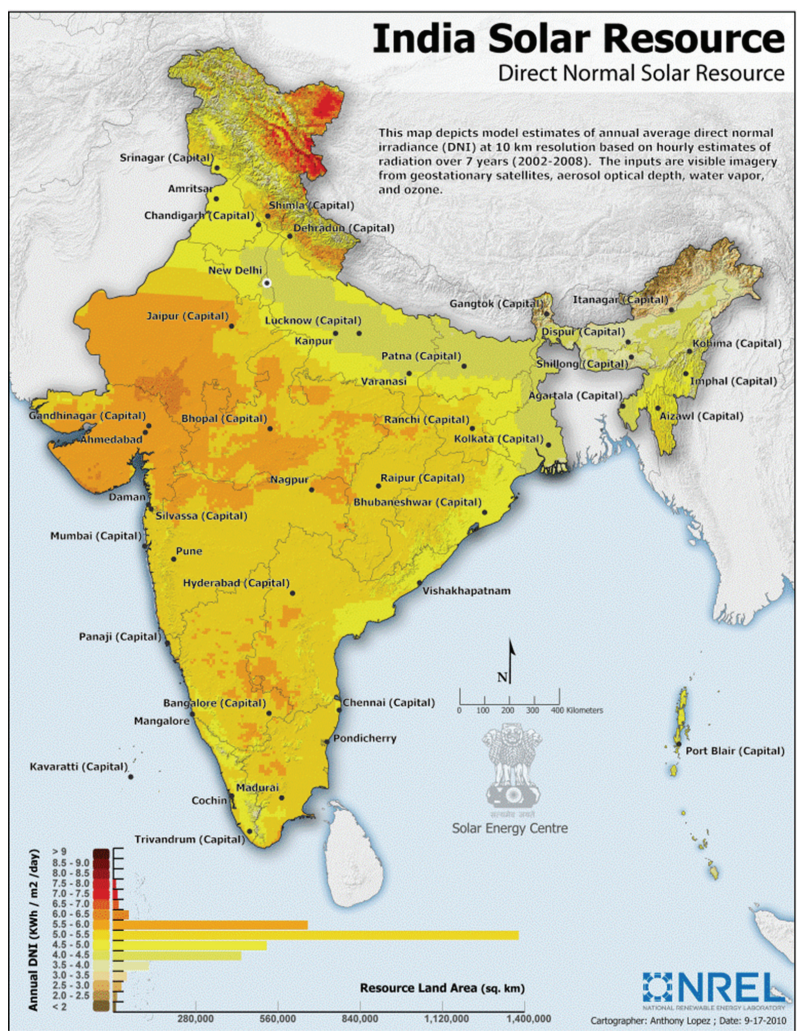

FIGURE 1: India's DNI resource at $10 \mathrm{~km}$ resolution (source: NREL $[8])$.

global radiation varies from 1600 to $2200 \mathrm{kWh} / \mathrm{m}^{2}$ [6], which is typical of the tropical and subtropical regions. NREL recently released $10 \mathrm{~km}$ resolution solar resource maps for India based on the SUNY satellite. Figure 1 shows the annual average direct normal irradiance (DNI) across India, which illustrates that most areas of the country have greater than $5 \mathrm{kWh} / \mathrm{m}^{2} /$ day of DNI. This image shows the areas of highest resource occurring in the state of Gujarat, Rajasthan, and the high-elevation Himalayan region. Figure 1 also shows large areas with annual average DNI greater than $5.5 \mathrm{kWh} / \mathrm{m}^{2} /$ day in the state of Madhya Pradesh, Chhattisgarh, and Maharashtra and smaller land areas with similar resources in several other states. This area could be further increased by the use of building-integrated PV. Though large-scale CSP has not yet been deployed in India; one study has estimated that this technology alone could generate 11,000 TWh per year for India (Table 1). In addition, it also offers huge potential for decentralized distributed electricity supply system, which can address the problem of electricity to a remote location with less/lower transmission losses.

In spite of huge solar energy potential, the portfolio of RE has developed in a very unique way in India, though, lately introduced wind power technology has left behind all the traditional RE technologies such as biomass, solar power, and SHP as shown in Figure 2. It also demonstrates that solar power has the maximum untapped potential as $97.9 \%$ of estimated potential of $50000 \mathrm{MW}$. And the overall untapped potential of $81.3 \%$ seeks immediate attention of policy makers
TABLE 1: India's estimated land area suitable for CSP development and generation potential [28].

\begin{tabular}{lcc}
\hline $\begin{array}{l}\text { DNI class } \\
\left(\mathrm{kWh} / \mathrm{m}^{2} / \text { year }\right)\end{array}$ & $\begin{array}{c}\text { Land area suitable for } \\
\text { CSP development }\left(\mathrm{km}^{2}\right)\end{array}$ & $\begin{array}{c}\text { CSP generating } \\
\text { potential (TWh/year) }\end{array}$ \\
\hline $2,000-2,099$ & 83,522 & 7,893 \\
$2,100-2,199$ & 11,510 & 1,140 \\
$2,200-2,299$ & 5,310 & 550 \\
$2,300-2,399$ & 7,169 & 774 \\
$2,400-2,499$ & 3,783 & 426 \\
$2,500-2,599$ & 107 & 13 \\
$2,600-2,699$ & 976 & 119 \\
$2,700-2,800+$ & 120 & 15 \\
\hline Total & 112,497 & 10,930 \\
\hline
\end{tabular}

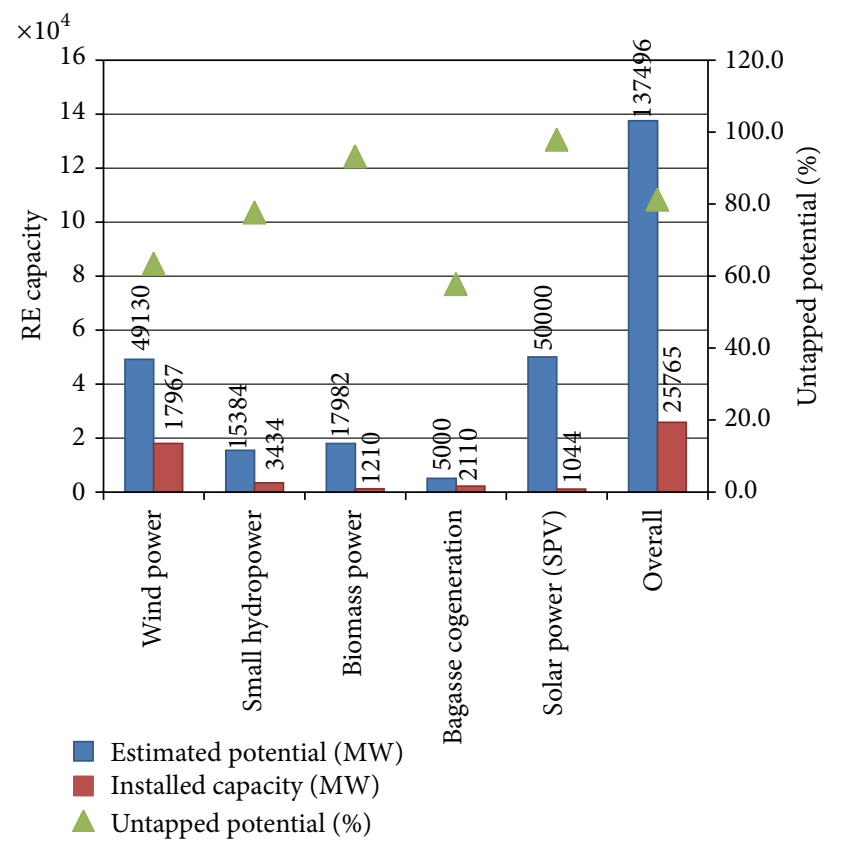

FIGURE 2: Development of overall RE portfolio as on 31st of August, 2012 (source: MNRE [9]).

for exploiting this available RE resource in a very efficient and effective manner to overcome the problem of electricity shortage.

\section{Development of Solar Electricity System under Different Policy Measures in India}

Government of India has recognized the importance of solar energy as one of the sustainable sources of energy under National Action Plan for Climate Change (NAPCC). NAPCC aims to derive $15 \%$ of its energy requirements from $\mathrm{RE}$ sources by the year 2020 [10]. Various policy measures, such as preferential tariff or fixed tariff or feed-in tariff (FiT), RPO, excise duty exemption, and soft loan, have been implemented to achieve the above-mentioned target. 
TABLE 2: State-wise solar RPO.

\begin{tabular}{|c|c|c|c|c|c|c|c|c|c|c|c|}
\hline \multirow{2}{*}{ State } & \multicolumn{11}{|c|}{ RPO (\%) } \\
\hline & FY 2012 & FY 2013 & FY 2014 & FY 2015 & FY 2016 & FY 2017 & FY 2018 & FY 2019 & FY 2020 & FY 2021 & FY 2022 \\
\hline Andhra Pradesh & 0.25 & 0.25 & 0.25 & 0.25 & 0.25 & 0.25 & & & & & \\
\hline Arunachal Pradesh & \multicolumn{11}{|c|}{ No regulation issued for RPO by the power department } \\
\hline Assam & 0.10 & 0.15 & 0.20 & 0.25 & & & & & & & \\
\hline Bihar & 0.25 & 0.25 & 0.50 & 0.75 & 1.00 & 1.25 & 1.50 & 1.75 & 2.00 & 2.50 & 3.00 \\
\hline Chhattisgarh & 0.25 & 0.50 & & & & & & & & & \\
\hline Delhi & 0.10 & 0.15 & 0.20 & 0.25 & 0.30 & 0.35 & & & & & \\
\hline JERC (Goa and UT) & 0.30 & 0.40 & & & & & & & & & \\
\hline Gujarat & 0.50 & 1.00 & & & & & & & & & \\
\hline Haryana & 0.00 & 0.05 & 0.75 & & & & & & & & \\
\hline Himachal Pradesh & 0.01 & 0.25 & 0.25 & 0.25 & 0.25 & 0.25 & 0.50 & 0.75 & 1.00 & 2.00 & 3.00 \\
\hline Jammu and Kashmir & 0.10 & 0.25 & & & & & & & & & \\
\hline Jharkhand & 0.50 & 1.00 & & & & & & & & & \\
\hline Karnataka & 0.25 & & & & & & & & & & \\
\hline Kerala & 0.25 & 0.25 & 0.25 & 0.25 & 0.25 & 0.25 & 0.25 & 0.25 & 0.25 & 0.25 & 0.25 \\
\hline Madhya Pradesh & 0.40 & 0.60 & 0.80 & 1.00 & & & & & & & \\
\hline Maharashtra & 0.25 & 0.25 & 0.50 & 0.50 & 0.50 & & & & & & \\
\hline Manipur & 0.25 & 0.25 & & & & & & & & & \\
\hline Mizoram & 0.25 & 0.25 & & & & & & & & & \\
\hline Meghalaya & 0.30 & 0.40 & & & & & & & & & \\
\hline Nagaland & 0.25 & 0.25 & & & & & & & & & \\
\hline Orissa & 0.10 & 0.15 & 0.20 & 0.25 & 0.30 & & & & & & \\
\hline Punjab & 0.03 & 0.07 & 0.13 & 0.19 & & & & & & & \\
\hline Rajasthan & 0.50 & 0.75 & 1.00 & & & & & & & & \\
\hline Sikkim & \multicolumn{11}{|c|}{ No regulation issued for RPO by the power department } \\
\hline Tamil Nadu & 0.05 & & & & & & & & & & \\
\hline Tripura & 0.10 & 0.10 & & & & & & & & & \\
\hline Uttarakhand & 0.03 & 0.05 & & & & & & & & & \\
\hline Uttar Pradesh & 0.50 & 1.00 & & & & & & & & & \\
\hline West Bengal & & & 0.25 & 0.30 & 0.40 & 0.50 & & & & & \\
\hline
\end{tabular}

Source: SERCs order on RPO regulations [29].

RPO is one of the tools which have been implemented by many countries to achieve their ambitious RE goals [11, 12]. In India, state electricity regulatory commissions (SERCs) determine the obligated entities, which generally include distribution companies, captive consumers, and any open-access users. Then these SERCs fix a certain proportion of electricity consumption as RPO targets for the above-mentioned obligated entities. Due to significant cost difference in solar and nonsolar RE technologies [13], most of the states have come up with their separate targets for solar electricity, as shown in Table 2. As per the National Tariff Policy, it is envisaged that the targets for solar RPO shall be $0.25 \%$ by $2012-2013$ extending to $3 \%$ by 2022 [14], but only the state of Bihar has followed it in its solar policy the way it is expected.

A study conducted by MNRE shows that most of the states could not install capacity of solar system as per their RPO compliance requirement for the year 2012-2013 as on 12th of November 2012 (Table 3). The states like Uttar Pradesh (422.7 MW), Haryana (172.2 MW), Maharashtra (151.3 MW), and so forth, are far away from their RPO target. However, states of Gujarat (488.3 MW), Rajasthan (82.3 MW), and Karnataka (61.1 MW) have already surpassed their RPO requirement. Other states like Madhya Pradesh, Orissa, Punjab, and Uttarakhand may soon exceed their targets.

However, when annual solar capacity requirement for RPO compliance is analyzed (Table 4), it is found that India would need $\sim 34,000 \mathrm{MW}$ of solar capacity to achieve the target of 3\% contribution from solar electricity till 2022. It means that set RPO targets are not sufficient to have a solar energy dominant RE development scenario [15]. The policy makers expect to fill this gap with states' solar energy promotion policies and programmes. Most of the states have come up with their solar energy specific policies which are discussed in detail in subsequent sections.

As far as state-wise installation is concerned, Table 5 clearly demonstrates that states of Gujarat (65.83\%) and Rajasthan (19.20\%) have major share in total installed capacity (1047.87 MW). Other states like Andhra Pradesh (23.75 MW) and Maharashtra (21.00 MW) also have significant contribution. However, it is quite remarkable that out of 
TABLE 3: Expected solar RPO requirement and compliance for 2012-2013 [30].

\begin{tabular}{|c|c|c|c|c|c|c|c|}
\hline \multirow[t]{2}{*}{ State } & $\begin{array}{l}\text { Projected } \\
\text { demand }^{*} \\
\quad(\mathrm{MU})\end{array}$ & $\begin{array}{c}\text { Solar RPO } \\
\text { target } \\
(2012-2013)\end{array}$ & $\begin{array}{c}\text { Solar RPO } \\
\text { target } \\
(2012-2013)\end{array}$ & $\begin{array}{c}\text { Capacity required } \\
\text { for meeting solar } \\
\text { RPO }\end{array}$ & $\begin{array}{c}\text { Total capacity tied } \\
\text { up as on } \\
31.11 .2012^{*}\end{array}$ & $\begin{array}{c}\text { Installed capacity } \\
\text { as on } 31.11 .2012\end{array}$ & $\begin{array}{l}\text { Gap to be fulfilled } \\
\text { in } 2012-2013\end{array}$ \\
\hline & 2012-2013 & $\%$ & MU & MW & MW & MW & MW \\
\hline Andhra Pradesh & 98,956 & 0.25 & 247.39 & 148.6 & 75.5 & 23.75 & 73.1 \\
\hline Assam & 6,810 & 0.15 & 10.21 & 6.1 & 5 & - & 1.14 \\
\hline Bihar & 15,272 & 0.75 & 114.54 & 68.8 & 0 & - & 68.82 \\
\hline Chhattisgarh & 21,174 & 0.50 & 105.87 & 63.6 & 29 & 4.00 & 34.61 \\
\hline Delhi & 28,598 & 0.15 & 42.90 & 25.8 & 2.525 & 2.53 & 23.25 \\
\hline JERC (Goa and UT) & 12,860 & 0.40 & 51.44 & 30.9 & 1.7 & 1.69 & 29.21 \\
\hline Gujarat & 79,919 & 1.00 & 799.19 & 480.2 & 968.5 & 689.81 & (488.3) \\
\hline Haryana & 40,167 & 0.75 & 301.25 & 181.0 & 8.8 & 7.80 & 172.2 \\
\hline Himachal Pradesh & 8,647 & 0.25 & 21.62 & 13.0 & 0 & - & 13.0 \\
\hline Jammu and Kashmir & r $\quad 14,573$ & 0.25 & 36.43 & 21.9 & 0 & - & 21.8 \\
\hline Jharkhand & 6,696 & 1.00 & 66.96 & 40.2 & 36 & 16.00 & 4.2 \\
\hline Karnataka & 65,152 & 0.25 & 162.88 & 97.9 & 159 & 14.00 & (61.1) \\
\hline Kerala & 21,060 & 0.25 & 52.65 & 31.6 & 0.025 & 0.03 & 31.6 \\
\hline Madhya Pradesh & 53,358 & 0.60 & 320.15 & 192.3 & 213.21 & 7.25 & (20.9) \\
\hline Maharashtra & 150,987 & 0.25 & 377.47 & 226.8 & 75.5 & 21.00 & 151.3 \\
\hline Manipur & 608 & 0.25 & 1.52 & 0.9 & 0 & - & 0.9 \\
\hline Mizoram & 418 & 0.25 & 1.04 & 0.6 & 0 & - & 0.6 \\
\hline Meghalaya & 2,154 & 0.40 & 8.62 & 5.2 & 0 & - & 5.2 \\
\hline Nagaland & 596 & 0.25 & 1.49 & 0.9 & 0 & - & 0.9 \\
\hline Orissa & 24,284 & 0.15 & 36.43 & 21.9 & 54 & 13.00 & (32.1) \\
\hline Punjab & 48,089 & 0.07 & 33.66 & 20.2 & 51.825 & 9.33 & (31.6) \\
\hline Rajasthan & 55,057 & 0.75 & 412.93 & 248.1 & 330.4 & 201.15 & (82.3) \\
\hline Tamil Nadu & 91,441 & 0.05 & 45.72 & 27.5 & 18.105 & 17.05 & 9.4 \\
\hline Tripura & 1,010 & 0.10 & 1.01 & 0.6 & 0 & - & 0.6 \\
\hline Uttarakhand & 11,541 & 0.05 & 5.77 & 3.5 & 5.05 & 5.05 & (1.6) \\
\hline Uttar Pradesh & 85,902 & 1.00 & 859.02 & 516.1 & 93.375 & 12.38 & 422.7 \\
\hline West Bengal & 41,896 & 0.25 & 104.74 & 62.9 & 52.05 & 2.05 & 10.9 \\
\hline & & & & $2,537.2$ & $2,179.59$ & $1,047.84$ & \\
\hline
\end{tabular}

Source: CEA base data for 2011-2012 and escalated for 2012-2013 based on 18th EPS escalation rates for the same period; ${ }^{*}$ based on the data provided by NVVN, state agencies, and project developers. Italic numbers are the surplus capacity built-up.

26 states 19 have shown their presence in the class of solar India in spite of relatively high generation cost (Table 5). It is expected that when cost will reduce with increasing capacity, states which have low or no capacity may contribute significantly.

Moreover, Table 6 shows that the installed capacity (1047.87 MW) under various policy measures is dominated by state policies $(65.83 \%)$ which is in particular the success mantra of state of Gujarat $(689.81 \mathrm{MW})$. The peculiarity of theses installation scenarios should be taken into account while devising the policy for future.

3.1. Development under JNNSM. To achieve the long-term goal of NAPCC, Jawaharlal Nehru National Solar Mission (JNNSM) was launched on 11th January, 2010, with an objective to maximize generation of power from solar energy. Thus, it also constitutes a major contribution by India to the global effort to meet the challenges of climate change. The immediate aim of the mission is to focus on setting up an enabling environment for solar technology penetration in the country both at a centralized and decentralized level [16].

Table 7 shows JNNSM's targets with time line. The first phase (up to March 2013) focuses on promoting off-grid systems including hybrid systems to serve the population which is located in remote areas. For the first phase modest targets were set under the expectation that this development would bring down costs under enabling framework and support for entrepreneurs to develop markets. This cost reduction would help in creating conducive environment to achieve the ambitious targets of the second phase (2013-17) and third phase (2017-2022). 
TABLE 4: Solar power capacity requirement by $2022[31]$ and $[15]^{\#}$.

\begin{tabular}{lccccc}
\hline Year & $\begin{array}{c}\text { Energy demand } \\
(\mathrm{MU})^{*} \\
(A)\end{array}$ & $\begin{array}{c}\text { Solar RPO }(\%) \\
(B)\end{array}$ & $\begin{array}{c}\text { Solar energy requirement (MU) } \\
\text { for RPO compliance } \\
(A \times B)\end{array}$ & $\begin{array}{c}\text { Solar capacity requirement } \\
\text { for RPO compliance } \\
(\mathrm{MW})\end{array}$ & $\begin{array}{c}\text { Solar capacity requirement } \\
\text { for solar dominant scenario } \\
(\mathrm{MW})^{\#}\end{array}$ \\
\hline $2011-2012$ & 953,919 & 0.25 & 2,385 & 1,433 & 514 \\
$2012-2013$ & $1,022,287$ & 0.25 & 2,556 & 1,536 & 2414 \\
$2013-2014$ & $1,095,555$ & 0.50 & 5,478 & 3,291 & 5414 \\
$2014-2015$ & $1,174,074$ & 0.75 & 8,806 & 5,291 & 8414 \\
$2015-2016$ & $1,258,221$ & 1.00 & 12,582 & 7,560 & 12414 \\
$2016-2017$ & $1,348,399$ & 1.25 & 16,855 & 10,127 & 17490 \\
$2017-2018$ & $1,443,326$ & 1.75 & 25,258 & 15,176 & 22490 \\
$2018-2019$ & $1,544,936$ & 2.25 & 34,761 & 20,885 & 28490 \\
$2019-2020$ & $1,653,700$ & 2.50 & 41,343 & 24,839 & 35490 \\
$2020-2021$ & $1,770,120$ & 2.75 & 48,678 & 29,247 & - \\
$2021-2022$ & $\mathbf{1 , 8 9 4 , 7 3 6}$ & $\mathbf{3 . 0 0}$ & $\mathbf{5 6 , 8 4 2}$ & 34,152 & - \\
\hline
\end{tabular}

Note: * based on the National Electricity Plan for Generation January 2012.

${ }^{\#}$ Source: WISE, Pune [15].

TABLE 5: State-wise distribution of installed solar capacity as on 31st of November, 2012 [32].

\begin{tabular}{|c|c|c|c|c|c|c|c|c|}
\hline \multirow{2}{*}{ Sr. no. } & \multirow{2}{*}{ States } & \multicolumn{7}{|c|}{ Installed capacity (MW) } \\
\hline & & JNNSM & State policy & RPSSGP/GBI scheme & REC & Other & Total & $\%$ of total \\
\hline 1 & Andhra Pradesh & 12.00 & - & 9.75 & - & 2.00 & 23.75 & 2.27 \\
\hline 2 & Arunachal Pradesh & - & - & - & - & 0.03 & 0.03 & 0.00 \\
\hline 3 & Chhattisgarh & - & - & 4.00 & - & - & 4.00 & 0.38 \\
\hline 4 & Delhi & - & - & - & - & 2.53 & 2.53 & 0.24 \\
\hline 5 & Goa and UT & - & - & - & - & 1.69 & 1.69 & 0.16 \\
\hline 6 & Gujarat & - & 689.81 & - & - & - & 689.81 & 65.83 \\
\hline 7 & Haryana & - & - & 7.80 & - & - & 7.80 & 0.74 \\
\hline 8 & Jharkhand & - & - & 16.00 & - & - & 16.00 & 1.53 \\
\hline 9 & Karnataka & 5.00 & - & - & - & 9.00 & 14.00 & 1.34 \\
\hline 10 & Kerala & - & - & - & - & 0.03 & 0.03 & 0.00 \\
\hline 11 & Madhya Pradesh & - & - & 5.25 & 2.00 & - & 7.25 & 0.69 \\
\hline 12 & Maharashtra & 11.00 & - & 5.00 & - & 5.00 & 21.00 & 2.00 \\
\hline 13 & Orissa & 5.00 & - & 7.00 & - & 1.00 & 13.00 & 1.24 \\
\hline 14 & Punjab & 2.00 & - & 6.00 & - & 1.33 & 9.33 & 0.89 \\
\hline 15 & Rajasthan & 137.50 & - & 10.00 & 2.75 & 50.90 & 201.15 & 19.20 \\
\hline 16 & Tamil Nadu & 5.00 & - & 5.00 & - & 7.05 & 17.05 & 1.63 \\
\hline 17 & Uttarakhand & - & - & 5.00 & - & 0.05 & 5.05 & 0.48 \\
\hline 18 & Uttar Pradesh & 5.00 & - & 7.00 & - & 0.38 & 12.38 & 1.18 \\
\hline \multirow[t]{2}{*}{19} & West Bengal & - & - & 一 & 一 & 2.05 & 2.05 & 0.20 \\
\hline & Total & 182.50 & 689.81 & 87.80 & 4.75 & 83.01 & 1047.87 & \\
\hline
\end{tabular}

TABLE 6: Policy-wise distribution of installed solar capacity as on 31st of November, 2012 [32].

\begin{tabular}{lcc}
\hline Projects & Capacity (MW) & \% of total \\
\hline Projects under JNNSM & 182.5 & 17.42 \\
Projects under the state policy & 689.81 & 65.83 \\
Projects under RPSSGP/GBI scheme & 87.8 & 8.38 \\
Projects under REC scheme & 4.75 & 0.45 \\
Other projects & 83.01 & 7.92 \\
\hline Total & 1047.87 \\
\hline
\end{tabular}


TABLE 7: JNNSM targets with timeline [16].

\begin{tabular}{lccr}
\hline & $2010-2013$ & $2013-2017$ & $2017-2022$ \\
\hline $\begin{array}{l}\text { Utility grid power } \\
\text { including roof top (MW) }\end{array}$ & $1000-2000$ & $4000-10000$ & 20000 \\
$\begin{array}{l}\text { Off-grid solar applications } \\
\text { (MW) }\end{array}$ & 200 & 1000 & 2000 \\
Solar collectors (sq. m) & 7 million & 15 million & 20 million \\
\hline
\end{tabular}

TABLE 8: Technology-wise capacity targets under JNNSM Phase II (MW) [17].

\begin{tabular}{lcccc}
\hline Segment & Share $(\%)$ & Capacity & Central scheme & State scheme \\
\hline Solar PV & 70 & 6300 & 2520 & 3780 \\
Solar thermal & 30 & 2700 & 1080 & 1620 \\
\hline Total & & 10000 & 3600 & 5400 \\
\hline
\end{tabular}

TABLE 9: Floor and forbearance prices for RECs $\left(\right.$ Rs. $^{2} /$ REC) ${ }^{*}$.

\begin{tabular}{lcr}
\hline & REC prices till 2011 & REC prices for 2012-2015 \\
\hline Forbearance price & 17000 & 13400 \\
Floor price & 12000 & 9300 \\
\hline
\end{tabular}

${ }^{*} 1 \mathrm{REC}=1 \mathrm{MWh}$.

${ }^{2} 1$ USD $=$ INR 54.5 as of September 20, 2012.

TABLE 10: Solar REC trading details from IEX [33].

\begin{tabular}{|c|c|c|c|c|c|c|}
\hline Year & Month & $\begin{array}{c}\text { Buy bids } \\
\text { (REC) }\end{array}$ & $\begin{array}{l}\text { Sell bids } \\
\text { (REC) }\end{array}$ & $\begin{array}{c}\text { Cleared volume } \\
\text { (REC) }\end{array}$ & $\begin{array}{c}\text { Cleared price } \\
\text { (Rs/REC) }\end{array}$ & No. of participants \\
\hline \multirow{11}{*}{2011} & February & 11 & - & - & - & 1 \\
\hline & March & 30,001 & - & - & - & 3 \\
\hline & April & - & - & - & - & - \\
\hline & May & - & - & - & - & - \\
\hline & June & - & - & - & - & - \\
\hline & July & - & - & - & - & - \\
\hline & August & 1 & - & - & - & 1 \\
\hline & September & 7 & - & - & - & 4 \\
\hline & October & 1 & - & - & - & 1 \\
\hline & November & 43 & - & - & - & 2 \\
\hline & December & 495 & - & - & - & - \\
\hline \multirow{12}{*}{2012} & January & 2,635 & - & - & - & 10 \\
\hline & February & 582 & - & - & - & 9 \\
\hline & March & 5,782 & - & - & - & 26 \\
\hline & April & 289 & - & - & - & 9 \\
\hline & May & 1,637 & 149 & 5 & 13,000 & 16 \\
\hline & June & 9,489 & 541 & 336 & 12,750 & 17 \\
\hline & July & 8,554 & 419 & 93 & 12,800 & 11 \\
\hline & August & 1,728 & 310 & 129 & 12,850 & 13 \\
\hline & September & 1,317 & 1,094 & 735 & 12,500 & 23 \\
\hline & October & 1,263 & 864 & 820 & 12,680 & 19 \\
\hline & November & 1,458 & 758 & 733 & 12,720 & 21 \\
\hline & December & 1,608 & 977 & 931 & 12,620 & 36 \\
\hline
\end{tabular}


TABLE 11: Compilation of recently declared preferential tariffs for solar PV and solar thermal technologies [27].

\begin{tabular}{|c|c|c|c|c|}
\hline \multirow{2}{*}{ State/centre } & \multicolumn{2}{|l|}{ Solar PV } & \multicolumn{2}{|c|}{ Solar thermal } \\
\hline & Tariff (Rs/kWh) & Control period & Tariff (Rs/kWh) & Control period \\
\hline CERC & $\begin{array}{l}\text { Lev-10.39 } \\
\text { AD-1.04 } \\
\text { After AD-9.35 }\end{array}$ & FY 2012-2013 & $\begin{array}{l}\text { Lev-12.46 } \\
\text { AD-1.24 } \\
\text { After AD-11.22 }\end{array}$ & FY 2012-2013 \\
\hline Andhra Pradesh & $\begin{array}{l}17.91 \text { (without } \mathrm{AD} \text { ) } \\
14.95 \text { (with } \mathrm{AD} \text { ) }\end{array}$ & $\begin{array}{l}2010-2011 \text { and } \\
2011-2012\end{array}$ & $\begin{array}{l}15.31 \text { (without } \mathrm{AD} \text { ) } \\
12.85 \text { (with } \mathrm{AD} \text { ) }\end{array}$ & $\begin{array}{l}2010-2011 \text { and } \\
2012-2013\end{array}$ \\
\hline Bihar & $\begin{array}{l}10.9 \text { (without } \mathrm{AD} \text { ) } \\
09.85 \text { (with } \mathrm{AD} \text { ) }\end{array}$ & $\begin{array}{l}\text { Up to March 31, } \\
2015 \\
\text { commissioning }\end{array}$ & $\begin{array}{l}13.11 \text { (without } \mathrm{AD} \text { ) } \\
11.87 \text { (with } \mathrm{AD} \text { ) }\end{array}$ & $\begin{array}{l}\text { Up to March 31, } \\
2015 \\
\text { commissioning }\end{array}$ \\
\hline Gujarat & $\begin{array}{l}\text { For MW scale plants: } \\
\text { Jan 2012-March 2013: } 10.37 \\
\text { (without AD), } 09.28 \text { (with AD), } \\
\text { FY 2013-2014: } 9.64 \text { (without AD), } \\
8.63 \text { (with AD), } \\
\text { FY 2014-2015: } 8.97 \text { (without AD), } \\
\text { 8.03 (with AD) } \\
\text { For } k W \text { scale plants } \\
\text { Jan 2012-March } 2013: 12.44 \\
\text { (without AD), } 11.14 \text { (with AD), } \\
\text { FY 2013-2014: } 11.57 \text { (without } \\
\text { AD), 10.36 (with AD), } \\
\text { FY 2014-2015: } 10.76 \text { (without } \\
\text { AD), 9.63 (with AD) }\end{array}$ & $\begin{array}{l}\text { Jan 29, } 2012 \text { to } \\
\text { March 31, } 2015\end{array}$ & $\begin{array}{l}12.91 \text { (without } \mathrm{AD} \text { ) } \\
11.55 \text { (with } \mathrm{AD} \text { ) }\end{array}$ & $\begin{array}{l}\text { Jan 29, } 2012 \text { to } \\
\text { March 31, } 2015\end{array}$ \\
\hline Haryana & $\begin{array}{l}9.18 \text { (SPV crystalline) } \\
8.90 \text { (SPV thin film) }\end{array}$ & $\begin{array}{l}3 \text { years (till FY } \\
2013-\text { FY 2015) }\end{array}$ & 12.17 & $\begin{array}{l}3 \text { years (till FY } \\
2013-\text { FY 2015) }\end{array}$ \\
\hline Karnataka & $\begin{array}{l}14.5 \text { (including rooftop and small } \\
\text { solar PV plants) }\end{array}$ & $\begin{array}{l}\text { Up to March 31, } \\
2013 \\
\text { commissioning }\end{array}$ & 11.35 & $\begin{array}{l}\text { Up to March 31, } \\
2013 \\
\text { commissioning }\end{array}$ \\
\hline Kerala & 15.18 (including incentives) & $\begin{array}{l}\text { For projects } \\
\text { commissioned } \\
\text { before Dec. 31, } \\
2009\end{array}$ & & \\
\hline Madhya Pradesh & $\begin{array}{l}10.44 \text { (capacity > } 2 \mathrm{MW}) \\
10.70 \text { (capacity up to } 2 \mathrm{MW} \text { ) }\end{array}$ & $\begin{array}{l}\text { Aug } 2012 \text { to March } \\
2014\end{array}$ & 12.65 & $\begin{array}{l}\text { Aug } 2012 \text { to March } \\
2014\end{array}$ \\
\hline Maharashtra & $\begin{array}{l}\text { Lev-11.16 (11.66-RT and SSPGP) } \\
\text { AD-1.65 (1.65) } \\
\text { After AD-9.51 (10.01) }\end{array}$ & $\begin{array}{l}\text { FY 2010-2014 (5 } \\
\text { years from the date } \\
\text { of } \\
\text { commencement) }\end{array}$ & $\begin{array}{l}\text { Lev-13.44 } \\
\text { AD-1.97 } \\
\text { After AD-11.47 }\end{array}$ & $\begin{array}{l}\text { FY 2010-2014 (5 } \\
\text { years from the date } \\
\text { of } \\
\text { commencement) }\end{array}$ \\
\hline Orissa & $\begin{array}{l}\text { Lev-17.80 } \\
\text { AD-3.03 } \\
\text { After AD-14.77 }\end{array}$ & $\begin{array}{l}\text { Plant } \\
\text { commissioned in } \\
\text { FY (2012-2013) } \\
\text { onwards }\end{array}$ & $\begin{array}{l}\text { Lev-14.73 } \\
\text { AD-2.41 } \\
\text { After AD-12.32 }\end{array}$ & $\begin{array}{l}\text { Plant } \\
\text { commissioned in } \\
\text { FY (2012-2013) } \\
\text { onwards }\end{array}$ \\
\hline Punjab & $\begin{array}{l}\text { Lev-10.39 } \\
\text { AD-1.04 } \\
\text { After AD-9.35 }\end{array}$ & $\begin{array}{l}\text { FY 2012-2016 ( } 5 \\
\text { years from the } \\
\text { notification of } \\
\text { order) }\end{array}$ & $\begin{array}{l}\text { Lev-12.46 } \\
\text { AD-1.24 } \\
\text { After AD-11.22 }\end{array}$ & FY 2012-2016 \\
\hline Rajasthan & $\begin{array}{l}9.63 \text { (plant commissioned by 31st } \\
\text { of March 2014) } \\
\text { with AD-10.45 } \\
9.63 \text { (roof top and SSPG } \\
\text { commissioned by 31st March } \\
\text { 2014) }\end{array}$ & & $\begin{array}{l}11.95 \text { (without AD) } \\
\text { With AD-10.45 } \\
11.95 \text { (small solar thermal power } \\
\text { gen commissioned by 31st of } \\
\text { March 2015) }\end{array}$ & FY 2012-2013 \\
\hline Tamil Nadu & $\begin{array}{l}\text { Lev-18.45 } \\
\text { AD-4.11 } \\
\text { After AD-14.34 } \\
\end{array}$ & $\begin{array}{l}\text { FY 2010-2011 (till } \\
31 / 05 / 12)\end{array}$ & $\begin{array}{l}\text { Lev-15.51 } \\
\text { AD-3.35 } \\
\text { After AD-12.16 }\end{array}$ & $\begin{array}{l}\text { FY 2010-2011 (till } \\
31 / 05 / 12)\end{array}$ \\
\hline
\end{tabular}


TABLE 11: Continued.

\begin{tabular}{|c|c|c|c|c|}
\hline \multirow{2}{*}{ State/centre } & \multicolumn{2}{|l|}{ Solar PV } & \multicolumn{2}{|c|}{ Solar thermal } \\
\hline & Tariff (Rs/kWh) & Control period & Tariff (Rs/kWh) & Control period \\
\hline Uttarakhand & $\begin{array}{l}\text { Lev-17.70 } \\
\text { AD-1.65 } \\
\text { After AD-16.05 }\end{array}$ & $\begin{array}{l}\text { FY 2009-2012 ( } 3 \\
\text { years from the } \\
\text { commencement of } \\
\text { these regulations) }\end{array}$ & $\begin{array}{l}\text { Lev-12.95 } \\
\text { AD-1.15 } \\
\text { After AD-11.80 }\end{array}$ & $\begin{array}{l}\text { FY 2009-2012 ( } 3 \\
\text { years from the } \\
\text { commencement of } \\
\text { these regulations) }\end{array}$ \\
\hline Uttar Pradesh & $\begin{array}{l}15 \text { (commissioned by Dec 2011, } \\
\text { not covered under GOI incentive } \\
\text { scheme) }\end{array}$ & $\begin{array}{l}\text { FY 2010-2014 (5 } \\
\text { years) }\end{array}$ & $\begin{array}{l}13 \text { (commissioned by Dec 2011, } \\
\text { not covered under GOI incentive } \\
\text { scheme) }\end{array}$ & $\begin{array}{l}\text { FY 2010-2014 (5 } \\
\text { years) }\end{array}$ \\
\hline West Bengal & $\begin{array}{l}10 \text { (capacity ranging } 100 \mathrm{KW} \text { to } \\
2 \mathrm{MW} \text { availing GBI) } \\
10 \text { (grid connected plant not } \\
\text { eligible for any incentive and } \\
\text { commissioned up to 2012-2013) } \\
10 \text { (projects commissioned after } \\
\text { FY } 2012 \text { till FY 2015) }\end{array}$ & $\begin{array}{l}\text { FY 2013-2017 (5 } \\
\text { years) }\end{array}$ & NA & NA \\
\hline
\end{tabular}

To attract the investors, JNNSM devises various incentive instruments such as RE Voucher/Stamp, Capital Subsidy, Interest Subsidy, Viability Gap Funding (VGF), and Green Energy Bonds. However, the capacity built is only $182.5 \mathrm{MW}$ which is concentrated around few states like Rajasthan (137.5 MW), Andhra Pradesh (12 MW), Maharashtra (11 MW), and so forth (refer: Table 5) under first phase (as on 31st of November, 2012). This seeks immediate attention of policy makers for reviewing their implementation strategy.

Recently announced JNNSM second phase policy mandate [17] includes the learning from the initial setback. It gives more weightage to state scheme $(5400 \mathrm{MW})$ than central scheme (3600 MW) for target capacity installation (Table 8). It also focuses more on VGF for incentivizing the projects.

To make the efforts more concentrated and specific, in a recent initiative, MNRE has given approval to the development of 54 solar cities [18]. As of now (January 21, 2013), 8 master plans have been approved for eight cities-Agra and Moradabad from Uttar Pradesh; Thane and Kalyan-Dombivli from Maharashtra; Indore from Madhya Pradesh; Kohima from Nagaland; and Aizawl from Mizoram and Chandigarh [18]. Thus by reducing scale, MNRE expects better solar capacity development in the near future.

3.2. Development under REC. The REC mechanism is a market-based instrument to promote renewable sources of energy and development of market in electricity, leading to the sustainable development of the country. REC mechanism has been designed to address the mismatch between availability of renewable electricity and the requirement of the obligated entities to meet their RPO by purchasing green attributes of RE remotely located in the form of REC. The implementation issues as well as institutional framework for India have been discussed in detail by Singh $[19,20]$ and MNRE [21]. Goyal and Jha [22] discussed in detail the framework to promote RE through a framework which puts into place Renewable Purchase Obligation (RPO) mechanism.

In India, RECs trading began in March 2011, on the platform of Indian Energy Exchange (IEX) and Power Exchange of India (PXI). Though during initial period it could not perform as per expectation, but the performance has been improving during the last few months, as shown in Table 10. But still due to large unmet demand, solar REC price has been hovering around the forbearance price (Table 9) of Rs. 13400/ REC. However, a recent notification by MNRE [23] which clarified state agencies for allowing use of solar REC to nonsolar RPO if it is above its minimum prescribed limit may increase the liquidity of solar REC market which is expected to decrease the price of it.

Though the capacity installed under this mechanism is only $4.75 \mathrm{MW}$ (Table 6), but capacity registered under this mechanism has reached close to $20 \mathrm{MW}$ [24]. And the present high price of REC holds bright prospects for this newly launched incentive scheme.

3.3. Development under RPSSGP/GBI Scheme. Rooftop Photovoltaic (PV) and Small Solar Power Generation Programme (RPSSGP) is a Generation-Based Incentives (GBIs) programme of the Ministry of New and Renewable Energy (MNRE) under the JNNSM for rooftop and other small solar plants. As of now, the installed capacity of $87.8 \mathrm{MW}$, which is well distributed across many states (Table 5), has been developed under this scheme. Due to limited access to smart grid/ net metering connectivity, this incentive has not yet been harnessed to its fullest by the developers.

3.4. Development under the State Policy. Most of the states have been using preferential tariffs as a tool to promote solar energy in their states (Table 11). But, due to huge financial burden, states of Gujarat and Andhra Pradesh have switched their focus from preferential tariff to REC market in their recently announced solar policies $[25,26]$. Moreover, state of Gujarat has also made provision for sharing Clean Development Mechanism (CDM) benefits to developers, starting from $100 \%$ in first year after commissioning, and thereafter reducing it by $10 \%$ every year till the sharing becomes equal $(50: 50)$ between the developers and the consumers [25].

Likewise several states have also come up with many other encouraging policies like Accelerated Depreciation (AD) on capital investment, soft loan for financing, reduced or no 
transmission and wheeling charges, no cross subsidy surcharge for open-access transactions, reduced or no intra-state Availability-Based Tariff (ABT), nonapplicability of merit order dispatch principles, exemption from electricity tax, tax concessions, refund of stamp duty and registration charges paid for land purchase, single window clearance, faster power evacuation approval, and so forth [25-27].

\section{Conclusion}

The study concludes that though JNNSM first phase could not perform up to the expectations, the state level policies have been doing well to fill the gap. The recently announced JNNSM Phase II target composition of $3600 \mathrm{MW}$ for central and $5400 \mathrm{MW}$ for states, changes in policies and schemes in light of the experience of Phase I, and development of 54 solar cities are likely to impact the National Solar Mission performance and enhance the overall visibility of solar-based electricity generation and utilization.

\section{Abbreviations}

CDM: Clean development mechanism

CSP: Concentrated solar power

DNI: Direct normal irradiance

FiT: $\quad$ Feed-in tariff

GBIs: Generation-based incentives

GoI: $\quad$ Government of India

IEX: Indian energy exchange

IMD: Indian meteorological department

JNNSM: Jawaharlal Nehru national solar mission

MNRE: Ministry of new and renewable energy

NAPCC: National action plan for climate change

NREL: National renewable energy laboratory

PV: $\quad$ Photovoltaic

PXI: $\quad$ Power exchange of India

RE: $\quad$ Renewable energy

REC: $\quad$ Renewable energy certificate

RPO: Renewable purchase obligation

RPSSGP: Rooftop photovoltaic and small solar power generation programme

SERC: State electricity regulatory commission

SHP: $\quad$ Small hydropower

VGF: Viability gap funding.

\section{References}

[1] IEA, World Energy Outlook, International Energy Agency, Paris, France, 2011.

[2] GEA, Global Energy Assessment: Toward a Sustainable Future, Cambridge University Press, Cambridge, UK, 2012.

[3] REN21, “Renewables 2012," Global Status Report, REN21 Secretariat, Paris, France, 2012.

[4] IPCC, "Special Report on Renewable Energy Sources and Climate Change Mitigation," Cambridge University Press, Cambridge, UK, 2007.

[5] IEA, Energy Technology Perspectives, International Energy Agency, Paris, France, 2008.
[6] “Solar radiant Energy over India," India Meteorological Department, Ministry of Earth Sciences, Government of India, 2009.

[7] M. Pipattanasomporn, A study of remote area internet access with embedded power generation [Ph.D. thesis], Virginia Polytechnic Institute and State University, Alexandria, VA, USA, 2004.

[8] NREL, http://www.nrel.gov/international/ra_india.html, 2010.

[9] http://mnre.gov.in, 2012.

[10] GOI, "National Action Plan on Climate Change," Prime Minister's Council on Climate Change, Government of India (GOI), New Delhi, India, 2008, http://www.forumofregulators.gov.in/ Data/study/TOR_Incentive $\% 20$ structure $\% 20$ for $\% 20$ fulfillment \%20of\%20RPO.pdf.

[11] M. Ringel, "Fostering the use of renewable energies in the European Union: the race between feed-in tariffs and green certificates," Renewable Energy, vol. 31, no. 1, pp. 1-17, 2006.

[12] P. Menanteau, D. Finon, and M. L. Lamy, "Prices versus quantities: choosing policies for promoting the development of renewable energy," Energy Policy, vol. 31, no. 8, pp. 799-812, 2003.

[13] Global Trends in Renewable Energy Investment, Frankfurt School-UNEP Collaborating Centre for Climate \& Sustainable Energy Finance, 2012.

[14] http://www.mnre.gov.in/information/solar-rpo/, 2013.

[15] WISE, "Achieving 12\% green electricity by 2017," Tech. Rep., World Institute of Sustainable Energy, Pune, India, 2010.

[16] MNRE, Jawaharlal Nehru National Solar Mission (JNNSM), Ministry of New and Renewable Energy (MNRE), Government of India, New Delhi, India, 2010, http://www.mnre.gov.in/filemanager/UserFiles/mission_document_JNNSM.pdf.

[17] http://mnre.gov.in/file-manager/UserFiles/draft-jnnsmpd-2. pdf, 2013.

[18] http://www.pv-magazine.com/news/details/beitrag/indiaidentifies-54-solar-cities-releases-jnnsm-figures_100009617/ \#axzz2J5UVafsH, 2013.

[19] A. Singh, "Economics, regulation and implementation strategy for renewable energy certificates in India," India Infrastructure Report, Infrastructure Development in a Low Carbon Economy, Oxford University Press, New Delhi, India, 2010.

[20] A. Singh, "A market for renewable energy credits in the Indian power sector," Renewable and Sustainable Energy Reviews, vol. 13, no. 3, pp. 643-652, 2009.

[21] MNRE, "Report on development of conceptual framework for renewable energy certificate mechanism for India," Tech. Rep., Ministry of New and Renewable Energy (MNRE), New Delhi, India, 2009, Prepared by ABPS Infrastructure Advisory Private Limited.

[22] M. Goyal and R. Jha, "Introduction of renewable energy certificate in the Indian scenario," Renewable and Sustainable Energy Reviews, vol. 13, no. 6-7, pp. 1395-1405, 2009.

[23] MNRE, MNRE, Government of India (GoI), New Delhi, India, http://mnre.gov.in/file-manager/UserFiles/solar-rpo.pdf, 2012.

[24] http://mnre.gov.in/file-manager/UserFiles/september_month_ 2012_rerf.pdf, 2013.

[25] http://geda.gujarat.gov.in/policy_files/GERC\%20order\%20 Solar\%20Energy.pdf, 2012.

[26] http://bridgetoindia.com/archive/policy/Andhra-PradeshSolar-Solar-Policy-2012-Abstract.pdf, 2013.

[27] http://mnre.gov.in/information/renewable-energy-regulatory framework, 2013. 
[28] http://www.dlr.de/tt/desktopdefault.aspx/tabid-2893/ httpstatus-404/, 2011.

[29] MNRE, MNRE, Government of India (GoI), New Delhi, India, http://mnre.gov.in/file-manager/UserFiles/Solar\%20RPO/ state-wise-solar-RPO-targets.pdf, 2012.

[30] http://mnre.gov.in/file-manager/UserFiles/Solar\%20RPO/ Expected\%20Solar\%20RPO\%20requirement\%20and\%20 compliance\%20for\%202012-13.xlsx, 2013.

[31] http://mnre.gov.in/file-manager/UserFiles/Solar\%20RPO/ solar-RPO-requirement-by-2022.pdf, 2013.

[32] http://mnre.gov.in/file-manager/UserFiles/Solar\%20RPO/ State\%20-\%20wise\%20solar\%20installed\%20capacity\% 20break-up.xlsx, 2013.

[33] http://www.iexindia.com/Reports/RECData.aspx, 2013. 


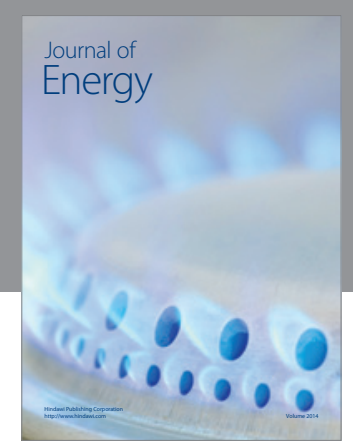

Journal of

Industrial Engineering
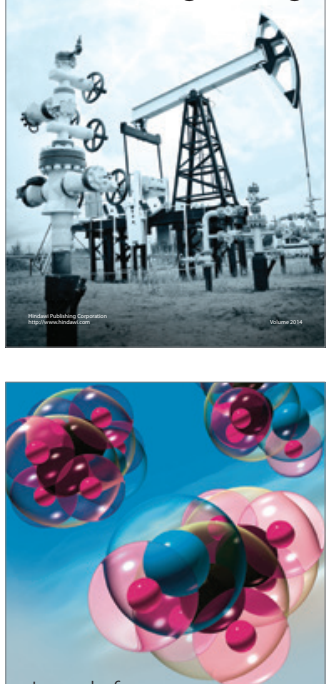

Fuels
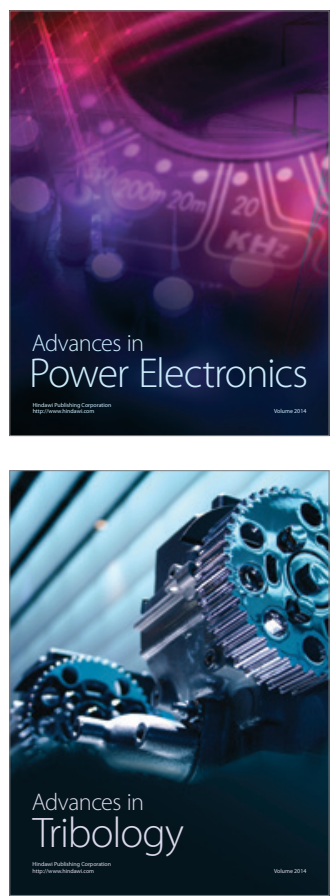

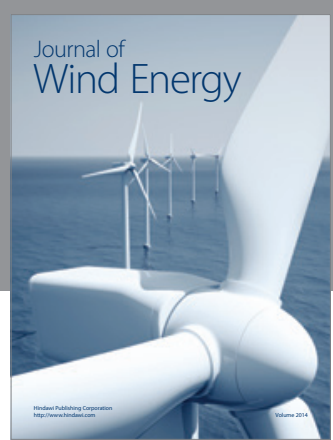

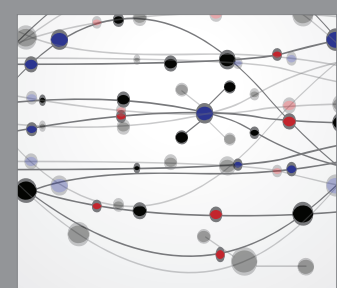

The Scientific World Journal

Submit your manuscripts at http://www.hindawi.com

Journal of

Structures
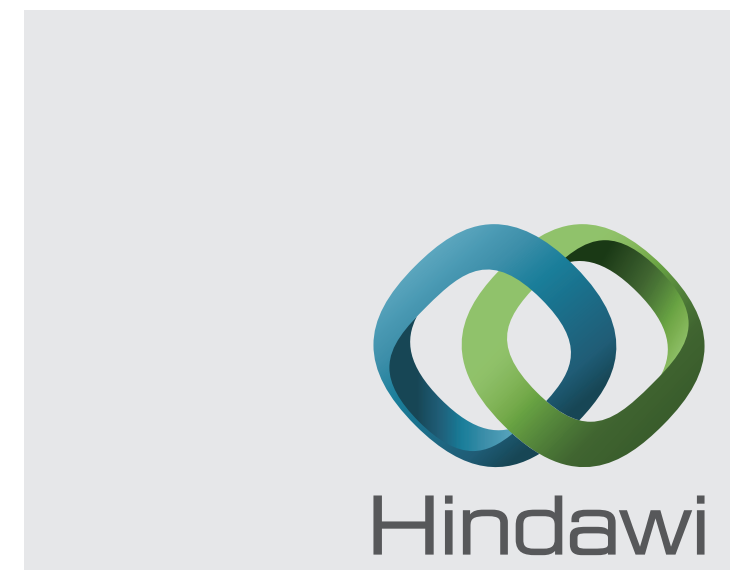

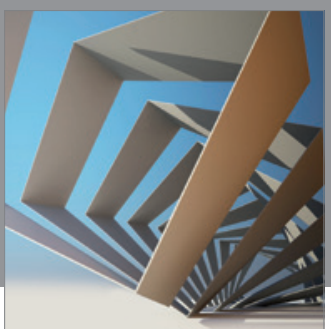

Rotating

Machinery
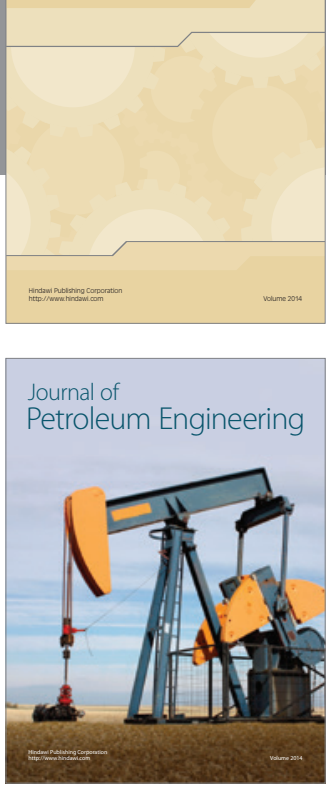

Journal of

Solar Energy
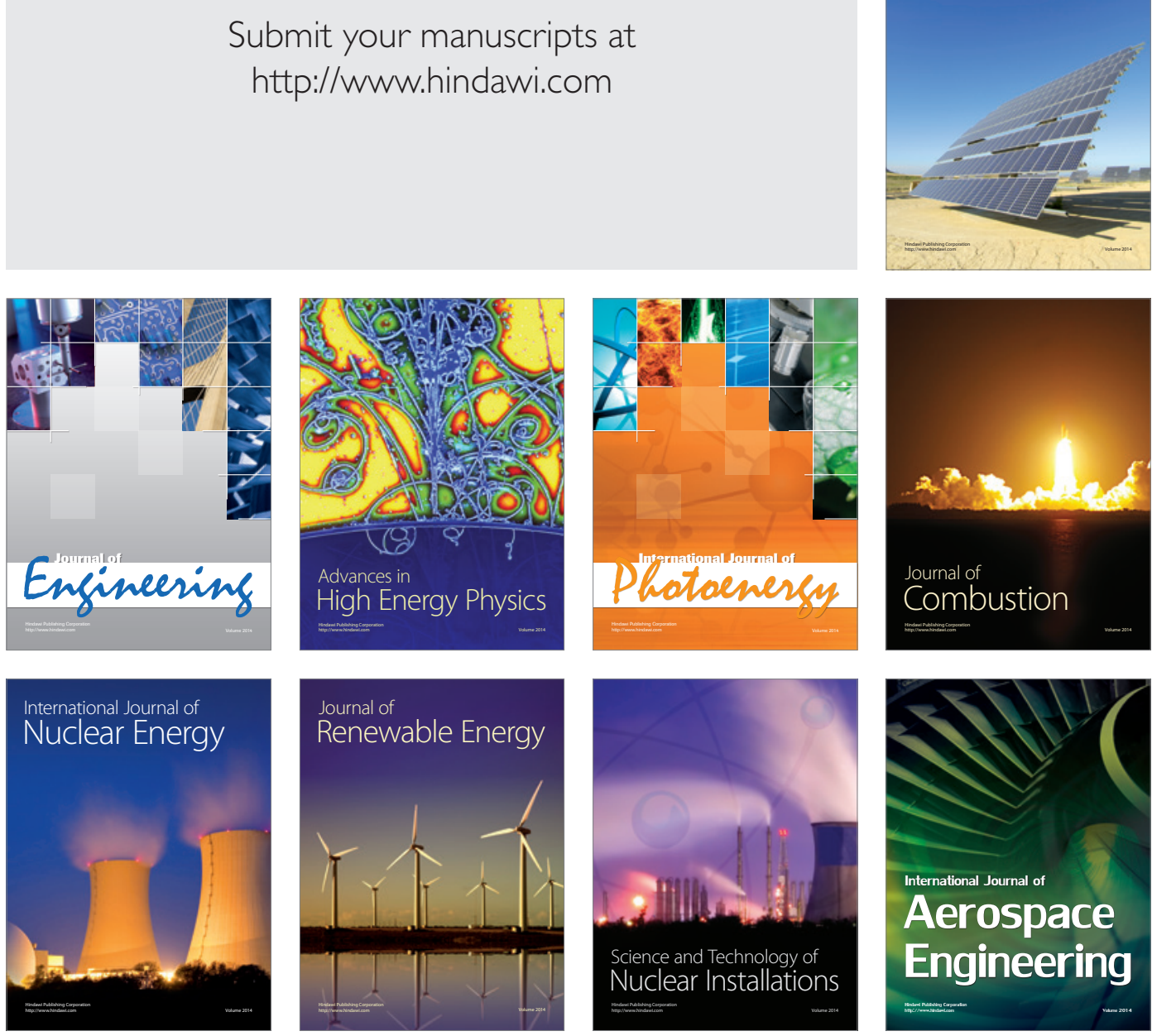